Nguyen Manh Hung, Tran Thi Loan (Hanoi Pedagogical Inst., Vietnam)

\title{
ON THE ASYMPTOTIC BEHAVIOUR OF SOLUTIONS OF THE FIRST INITIAL BOUNDARY-VALUE PROBLEMS FOR PARABOLIC EQUATIONS
}

\section{ПРО АСИМПТОТИЧНУ ПОВЕДІНКУ РОЗВ'ЯЗКІВ ПЕРШИХ ПОЧАТКОВИХ КРАЙОВИХ ЗАДАЧ ДЛЯ ПАРАБОЛГЧНИХ РІВНЯНЬ}

We consider the first initial boundary-value problem for a strongly parabolic system on infinite cylinder with non-smooth boundary. We prove some results on the existence, uniqueness and asymptotic behaviour of solutions as $t \rightarrow+\infty$.

Розглянуто першу початкову крайову задачу для сильно параболічної системи на нехінченному циліндрі із иегладко ме межен. Доведено деякі результати про існування, сдиність та асимттотичну поведінку роза'язкіз при $t \rightarrow+\infty$.

1. Introduction. The theory of general boundary-value problems for elliptic systems with smooth and non-smooth boundary is nearly completely considered in $[1,2]$. The strongly parabolic systems on a bounded set $\Omega_{T}=\Omega \times[0, T], \Omega \subset \mathbb{R}^{n}$, with nonsmooth boundary were studied in $[3,4]$. However, the boundary-value problem for a parabolic system on cylinder with non-smooth boundary has been rarely considered. O.A. Ladyzhenskaya [5] showed some sufficient conditions for the existence and uniqueness of a gencralized solution to the first boundary-value problem for strongly parabolic systems on a finite cylinder $\Omega_{T}$. The smoothness of generalized solutions for a parabolic equation of order 2 is considered in [6]. The general boundary-value problem on the finite cylinder for a parabolic system in the sense of Petrovski is studied in [7], where the author obtained some results on the uniqueness of a solution in the Sobolev weighted spacc. In [8], the second boundary-value problem for a parabolic equation of order 2 in infinite cylinder $\Omega_{\infty}=\Omega \times[-\infty,+\infty)$ is considered. Some results on the asymptotic behaviour of solutions as $t \rightarrow+\infty$ was shown in that paper.

Suppose that $\Omega \subset \mathbb{R}^{n}$ is a bounded domain with its boundary $\partial \Omega$. Let us introduce some notation: $\Omega_{b}=\Omega \times[0, b], \Gamma_{b}=\partial \Omega \times[0, b], \Omega_{\infty}=\Omega \times[0,+\infty), \Gamma_{\infty}=\partial \Omega \times$ $\times[0, \infty) ; \quad x=\left(x_{1}, \ldots, x_{n}\right) \in \Omega ; u(x, t)=\left(u_{1}(x, t), \ldots, u_{s}(x, t)\right)$ is a vector complex function; $\alpha=\left(\alpha_{1}, \ldots, \alpha_{n}\right), \alpha_{i} \in \mathbb{N},|\alpha|=\sum_{i=1}^{n} \alpha_{i} ; D^{\alpha}=\partial^{|\alpha|} / \partial x_{1}^{\alpha_{1}} \ldots \partial x_{n}^{\alpha_{n}}$, $\left|D^{\alpha} u\right|^{2}=\sum_{i=1}^{s}\left|D^{\alpha} u_{i}\right|^{2}, d x=d x_{1} \ldots d x_{n}, \quad u_{t^{j}}=\left(\partial^{j} u_{1} / \partial t^{j}, \ldots, \partial^{j} u_{s} / \partial t^{j}\right)$; $\stackrel{\circ}{C}^{\infty}(\Omega)$ is the set of infinitely differentiable functions having their supports compactly embedded in $\Omega ; H^{\ell}(\Omega)$ - the space consisting of functions $u(x)$ which have generalized derivatives $D^{\alpha} u_{i}$ belonging to $L_{2}(\Omega),|\alpha| \leq \ell, 1 \leq i \leq s$ and

$$
\|u\|_{H^{t}(\Omega)}^{2}=\sum_{|\alpha|=0}^{\ell} \int_{\Omega} \sum_{i=1}^{s}\left|D^{\alpha} u_{i}\right|^{2} d x<\infty \text {; }
$$

$\stackrel{\circ}{H}^{\ell}(\Omega)$ is the completion of $\stackrel{\circ}{C}^{\infty}(\Omega)$ in the norm of $H^{\ell}(\Omega) ; H^{\ell, k}\left(\Omega_{T}\right)$ is the space consisting of functions $u(x, t)$ such that $D^{\alpha} u_{i} \in L_{2}\left(\Omega_{T}\right), \partial^{j} u_{i} / \partial t^{j} \in L_{2}\left(\Omega_{T}\right)$, $|\alpha| \leq \ell, 1 \leq i \leq s, 1 \leq j \leq k$, with the norm 


$$
\|u\|_{H^{\varepsilon . k^{\prime}}\left(\Omega_{T}\right)}^{2}=\sum_{|\alpha|=0}^{\ell} \int_{\Omega_{T}}\left|D^{\alpha} u\right|^{2} d x d t+\sum_{j=1}^{k} \int_{\Omega_{T}}\left|u_{t j}\right|^{2} d x d t
$$

$H^{\ell, 0}\left(\Omega_{T}\right)$ is the space consisting of functions $u(x, t)$ with the norm

$$
\|u\|_{H^{\ell, 0}\left(\Omega_{T}\right)}^{2}=\sum_{|\alpha|=0}^{\ell} \int_{\Omega_{T}}\left|D^{\alpha} u\right|^{2} d x d t
$$

$\stackrel{\circ}{H}^{\ell, k}\left(\Omega_{T}\right)$ is the completion of $\stackrel{\circ}{C}^{\infty}\left(\Omega_{T}\right)$ in the norm of $H^{\ell, k}\left(\Omega_{T}\right) ; \stackrel{\circ}{\hat{H}^{m, 0}}\left(\Omega_{T}\right)=$ $=\left\{\eta(x, t) \in \stackrel{\circ}{H}^{m, 0}\left(\Omega_{T}\right): \eta(x, T)=0\right\}$.

Consider the differential operator

$$
L(x, t, D)=\sum_{|p|,|q|=1}^{m} D^{p} a_{p q}(x, t) D^{q}+\sum_{|p|=1}^{m} a_{p}(x, t) D^{p}+a(x, t),
$$

where $a_{p q}, a_{p}, a$ are $(s \times s)$-matrices, $a_{p q}=(-1)^{|p|+|q|} a_{q p}^{*}$, and their elements are bounded complex functions on $\bar{\Omega}_{\infty}$. Moreover, for every non-zero vector $\xi \in \mathbb{R}^{n}$ and $\eta \in \mathbb{C}^{s}$ we have

$$
\sum_{|p|,|q|=m} a_{p q}(x, t) \xi^{p} \xi^{q} \eta \bar{\eta}>0 \quad \forall(x, t) \in \bar{\Omega}_{\infty},
$$

where $\xi^{p}=\xi_{1}^{p_{1}} \ldots \xi_{n}^{p_{n}}, \xi^{q}=\xi_{1}^{q_{1}} \ldots \xi_{n}^{q_{n}}$.

We set

$$
B(u, v)=\sum_{|p|,|q|=1}^{m}(-1)^{|p|} \int_{\Omega} a_{p q} D^{q} u \overline{D^{p} v} d x
$$

and

$$
B_{1}(u, u)=B(u, u)+2 \operatorname{Re} \sum_{|p|=1}^{m} \int_{\Omega} a_{p} D^{p} u \bar{u} d x .
$$

The following lemma is proved in [9].

Lemma 1. If $a_{p q}$ satisfy (1) and $a_{p q}$ are continuous on $\bar{\Omega}_{\infty}$ whenever $|p|=$ $=|q|=m$ then there exist positive numbers $\mu_{0}, \mu_{1}, \lambda_{0}, \lambda_{1}$ such that

$$
\begin{aligned}
& (-1)^{m} B(u, u) \geq \mu_{0}\|u\|_{H^{m}(\Omega)}^{2}-\lambda_{0}\|u\|_{L^{2}(\Omega)}^{2}, \\
& (-1)^{m} B_{1}(u, u) \geq \mu_{1}\|u\|_{H^{m}(\Omega)}^{2}-\lambda_{1}\|u\|_{L^{2}(\Omega)}^{2}
\end{aligned}
$$

for all $u \in \stackrel{\circ}{H}^{m}(\Omega)$.

2. The existence of generalized solution. In this section we consider the existence and uniqueness of a solution of the problem

$$
(-1)^{m-1} L(x, t, D) u-u_{t}=f(x, t), \quad(x, t) \in \Omega_{\infty},
$$

such that

$$
u(x, 0)=0 \text { on } \Omega,
$$




$$
\left.\frac{\partial^{j} u}{\partial \nu^{j}}\right|_{\Gamma_{\infty}}=0, \quad j=0, \ldots, m-1,
$$

where $\nu$ is the unit outward normal to $\partial \Omega$.

Definition. A function $u(x, t)$ is called a generalized solution of $(4)-(6)$ in $H^{m, 1}\left(\Omega_{\infty}\right)$ if $u(x, t) \in \stackrel{\circ}{H}^{m, 1}\left(\Omega_{\infty}\right), u(x, 0)=0$ and for all $T>0, u(x, t)$ satisfies the following integnal identity on $\Omega_{T}$ :

$$
\begin{gathered}
(-1)^{m-1} \int_{\Omega_{T}}\left[\sum_{|p|,|q|=1}^{m}(-1)^{|p|} a_{p q} D^{q} u \overline{D^{p} \eta}+\sum_{|p|=1}^{m} a_{p} D^{p} u \bar{\eta}+a u \bar{\eta}\right] d x d t- \\
-\int_{\Omega_{\tau}} u_{t} \bar{\eta} d x d t=\int_{\Omega_{T}} f \bar{\eta} d x d t \quad \forall \eta \in \stackrel{\circ}{H}^{m, 0}\left(\Omega_{T}\right), \quad \eta(x, T)=0 .
\end{gathered}
$$

Theorem 1. If $a_{p q}$ are continuous on $\bar{\Omega}_{\infty}$ whenever $|p|=|q|=m$ and

$$
\left|\frac{\partial a_{p q}}{\partial t}, \frac{\partial a_{p}}{\partial t}\right| \leq \mu, \quad 1 \leq|p|, \quad|q| \leq m, \quad \mu=\text { const }
$$

then problem (4) - (6) has at most one generalized solution in $H^{m, 1}\left(\Omega_{\infty}\right)$.

Proof. Suppose that problem (4) $-(6)$ has two solutions $u_{1}, u_{2}$ and denote $u(x, t)=$ $=u_{1}(x, t)-u_{2}(x, t)$. For any $T>0$ and $b \in(0, T)$, we define $\eta(x, t)=\int_{b}^{t} u(x, \tau) d \tau$ for $0 \leq t \leq b$ and $\eta(x, t)=0$ for $b \leq t<T$. One can see that

$$
\eta_{t}(x, t)=u(x, t), \quad 0 \leq t \leq b, \quad \eta(x, t) \cdot \in \stackrel{\circ}{H}^{m, 0}\left(\Omega_{T}\right)
$$

and by identity (7), we have

$$
\begin{gathered}
(-1)^{m-1} \int_{\Omega_{b}}\left[\sum_{|p|,|q|=1}^{m}(-1)^{|p|} a_{p q} D^{q} \eta_{t} \overline{D^{p} \eta}+\sum_{|p|=1}^{m} a_{p} D^{p} \eta_{t} \bar{\eta}\right] d x d t+ \\
+(-1)^{m-1} \int_{\Omega_{b}} a \eta_{t} \bar{\eta} d x d t-\int_{\Omega_{b}} \eta_{t t} \bar{\eta} d x d t=0 .
\end{gathered}
$$

Let $a_{1}=a-(-1)^{m} \lambda_{1} I$, where $\lambda_{1}$ is a positive number satisfying (3). Thus,

$$
\begin{gathered}
(-1)^{m-1} \int_{\Omega_{b}}\left[\sum_{|p|,|q|=1}^{m}(-1)^{|p|} a_{p q} D^{q} \eta_{t} \overline{D^{p} \eta}+(-1)^{m} \lambda_{1} \eta_{t} \bar{\eta}+\right. \\
\left.+\sum_{|p|=1}^{m} a_{p} D^{p} \eta_{t} \bar{\eta}+a_{1} \eta_{t} \bar{\eta}\right] d x d t-\int_{\Omega_{\mathrm{b}}} \eta_{t t} \bar{\eta} d x d t=0 .
\end{gathered}
$$

Thus, the integration of the real parts of (9) gives

$2 \int_{\Omega_{b}}\left|\eta_{t}\right|^{2} d x d t+(-1)^{m}\left[\sum_{|p|,|q|=1}^{m}(-1)^{|p|} \int_{\Omega} a_{p q}(x, 0) D^{q} \eta(x, 0) \overline{D^{p} \eta(x, 0)} d x+\right.$ 


$$
\begin{aligned}
& \left.+2 \operatorname{Re} \sum_{|p|=1}^{m} \int_{\Omega} a_{p}(x, 0) D^{p} \eta(x, 0) \overline{\eta(x, 0)} d x\right]+\lambda_{1} \int_{\Omega}|\eta(x, 0)|^{2} d x= \\
& =(-1)^{m-1} \int_{\Omega_{b}} \sum_{|p|,|q|=1}^{m}(-1)^{|p|} \frac{\partial a_{p q}}{\partial t} D^{q} \eta \overline{D^{p} \eta} d x d t+ \\
& +(-1)^{m-1} 2 \operatorname{Re} \int_{\Omega_{b}}\left\{\sum_{|p|=1}^{m}\left[\frac{\partial a_{p}}{\partial t} D^{p} \eta \bar{\eta}+a_{p} D^{p} \eta \overline{\eta_{\bar{t}}}\right]+a_{1} \eta_{t} \bar{\eta}\right\} d x d t .
\end{aligned}
$$

Since $\frac{\partial a_{p q}}{\partial t}, \frac{\partial a_{p}}{\partial t}$ are bounded, by Cauchy inequality and (3) we obtain

$$
\left\|\eta_{t}\right\|_{L_{2}\left(\Omega_{b}\right)}^{2}+\|\eta(x, 0)\|_{H^{m}(\Omega)}^{2} \leq C(\varepsilon) \sum_{|p|=0}^{m} \int_{\Omega_{b}}\left|D^{p} \eta\right|^{2} d x d t+\varepsilon\left\|\eta_{t}\right\|_{L_{2}\left(\Omega_{b}\right)}^{2}
$$

and

$$
\|\eta(x, 0)\|_{H^{m}(\Omega)}^{2} \leq C \sum_{|p|=0}^{m} \int_{\Omega_{b}}\left|D^{p} \eta\right|^{2} d x d t .
$$

Setting

$$
v_{p}(x, t)=\int_{t}^{0} D^{p} u(x, \tau) d \tau, \quad 0<t<b, \quad J(t)=\sum_{|p|=0}^{m} \int_{\Omega}\left|v_{p}(x, t)\right|^{2} d x,
$$

we have

$$
(1-C b) J(b) \leq C \int_{0}^{b} J(t) d t, \quad b \in\left[0, \frac{1}{2} C\right] .
$$

By Gronwall-Bellman inequality, one has $J(t) \equiv 0$. Thus, (10), (11) lead to $\eta_{t} \equiv 0$, i.e., $u_{1} \equiv u_{2} \forall t \in[0,1 / 2 C]$. Using the same argument as before for functions $u_{1}$, $u_{2}$ on $[1 / 2 C, T]$, we can show that $u_{1} \equiv u_{2} \forall t \in[1 / 2 C, 1 / C]$. Continuing in this fashion, after finite number of steps, we can prove that $u_{1} \equiv u_{2} \forall t \in[0, T]$. Since $T>0$ is arbitrary, $u_{1} \equiv u_{2} \forall t \in[0, \infty)$. This completes the proof.

Now we prove the following theorem as a result on existence.

Theorem 2. Suppose that $a_{p q}$ are continuous on $\bar{\Omega}_{\infty}$ whenever $|p|=|q|=m$, there exists a $\lambda>0$, a function $B(t) \in L_{1}(0,+\infty)$ such that $f e^{\lambda t} \in L_{2}\left(\Omega_{\infty}\right)$ and

$$
\max \left\{\left|\frac{\partial a_{p q}}{\partial t}\right|,\left|a_{p}\right|,\left|a-(-1)^{m}\left(\lambda+\lambda_{0}\right) I\right|\right\} \leq B(t) \quad \forall(t, x) \in \Omega_{\infty},
$$

where $\lambda_{0}$ is determined from (2). Then problem (4)-(6) has a generalized solution $u(x, t) \in H^{m, 1}\left(\Omega_{\infty}\right)$ satisfying

$$
\|u\|_{H^{m, 1}\left(\Omega_{\infty}\right)}^{2} \leq C\left\|f e^{\lambda t}\right\|_{L_{2}\left(\Omega_{\infty}\right)}^{2} .
$$


Proof. Suppose that $\left\{\varphi_{k}(x)\right\}_{k=1}^{\infty} \subset \stackrel{\circ}{H}^{m}(\Omega)$ is orthonormal in $L_{2}(\Omega)$ and its linear closure is $\stackrel{\circ}{H}^{m}(\Omega)$. For each natural number $N$, let us consider the function

$$
u^{N}(x, t)=\sum_{k=1}^{N} C_{k}^{N}(t) \varphi_{k}(x),
$$

where $C_{k}^{N}(t)$ satisfies $C_{k}^{N}(0)=0$ and

$$
\begin{gathered}
\int_{\Omega}\left[u_{t}^{N} \overline{\varphi_{\ell}}+\sum_{|p|,|q|=1}^{m}(-1)^{m+|p|} a_{p q} D^{q} u^{N} \overline{D^{p} \varphi_{\ell}}+\lambda_{0} u^{N} \overline{\varphi_{\ell}}\right] d x+ \\
+(-1)^{m} \int_{\Omega}\left(\sum_{|p|=1}^{m} a_{p} D^{p} u^{N}+a_{0} u^{N}\right) \overline{\varphi_{\ell}} d x= \\
=-\int_{\Omega} f \overline{\varphi_{\ell}} d x, \quad \ell=1, \ldots, N,
\end{gathered}
$$

with $a_{0}=a-(-1)^{m} \lambda_{0} I$. Put $v^{N}(x, t)=u^{N}(x, t) e^{\lambda t}$. Multiplying (13) by $e^{\lambda t} \frac{d}{d t}\left(\overline{C_{\ell}^{N}(t) e^{\lambda t}}\right)$, then taking the sum in $\ell$ from 1 to $N$, one obtains

$$
\begin{gathered}
\int_{\Omega}\left|v_{t}^{N}\right|^{2} d x+\int_{\Omega} \sum_{|p|,|q|=1}^{m}\left[(-1)^{m+|p|} a_{p q} D^{q} v^{N} \overline{D^{p} v_{t}^{N}}+\lambda_{0} v^{N} \overline{v_{t}^{N}}\right] d x+ \\
+(-1)^{m} \int_{\Omega}\left[\sum_{|p|=1}^{m} a_{p} D^{p} v^{N} \overline{v_{t}^{N}}+\left(a_{0}-(-1)^{m} \lambda I\right) v^{N} \overline{v_{t}^{N}}\right] d x=-\int_{\Omega} f e^{\lambda t} \overline{v_{t}^{N}} d x .
\end{gathered}
$$

Since $a_{p q}=(-1)^{|p|+|q|} a_{q p}^{*}$, we have

$$
\begin{gathered}
2 \operatorname{Re}\left[\sum_{|p|,|q|=1}^{m}(-1)^{|p|} a_{p q} D^{q} v^{N} \overline{D^{p} v_{t}^{N}}+(-1)^{m} \lambda_{0} v^{N} \overline{v_{t}^{N}}\right]= \\
=\frac{\partial}{\partial t}\left(\sum_{|p|,|q|=1}^{m}(-1)^{|p|} a_{p q} D^{q} v^{N} \overline{D^{p} v^{N}}+(-1)^{m} \lambda_{0}\left|v^{N}\right|^{2}\right)- \\
-\sum_{|p|,|q|=1}^{m}(-1)^{|p|} \frac{\partial a_{p q}}{\partial t} D^{q} v^{N} \overline{D^{p} v^{N}} .
\end{gathered}
$$

By integrating the real parts of (14) in $t$ from 0 to $t$ and using (15), we get

$$
2 \int_{\Omega_{t}}\left|v_{t}^{N}\right|^{2} d x d t+
$$




$$
\begin{gathered}
+\int_{\Omega} \sum_{|p|,|q|=1}^{m}\left[(-1)^{m+|p|} a_{p q}(x, t) D^{q} v^{N}(x, t) \overline{D^{p} v^{N}(x, t)}+\lambda_{0}\left|v^{N}(x, t)\right|^{2}\right] d x= \\
=\int_{\Omega_{t}} \sum_{|p|,|q|=1}^{m}(-1)^{m+|p|} \frac{\partial a_{p q}}{\partial t} D^{q} v^{N} \frac{1}{D^{p} v^{N}} d x d t- \\
-(-1)^{m} 2 \operatorname{Re} \int_{\Omega_{L}}\left[\sum_{|p|=1}^{m} a_{p} D^{p} v^{N} \overline{v_{t}^{N}}+\bar{a} v^{N} \overline{v_{t}^{N}}\right] d x d t-2 \operatorname{Re} \int_{\Omega_{L}} f e^{\lambda t} \overline{v_{t}^{N}} d x d t,
\end{gathered}
$$

where $\bar{a}=\left(a_{0}-(-1)^{m} \lambda I\right)$.

By (2) and the Cauchy inequality, we obtain

$$
\begin{gathered}
2 \int_{\Omega_{t}}\left|v_{t}^{N}\right|^{2} d x d t+\mu_{0}\left\|v^{N}(x, t)\right\|_{H^{m}(\Omega)}^{2} \leq \\
\leq C_{1}(\varepsilon) \int_{0}^{t} B(t)\left\|v^{N}(x, t)\right\|_{H^{m}(\Omega)}^{2} d t+ \\
+\varepsilon \int_{\Omega_{t}}\left|v_{t}^{N}\right|^{2} d x d t+C_{2}(\varepsilon) \int_{0}^{t}\left\|f e^{\lambda t}\right\|_{L_{2}(\Omega)}^{2} d t .
\end{gathered}
$$

Therefore,

$$
\begin{gathered}
\int_{\Omega_{\iota}}\left|v_{t}^{N}\right|^{2} d x d t+\left\|v^{N}(x, t)\right\|_{H^{\prime \prime}(\Omega)}^{2} \leq \\
\leq C_{3} \int_{0}^{t} B(t)\left\|v^{N}(x, t)\right\|_{H^{m}(\Omega)}^{2} d t+C_{4} \int_{0}^{\infty}\left\|f e^{\lambda t}\right\|_{L_{2}(\Omega)}^{2} d t .
\end{gathered}
$$

So we get

$$
\left\|v^{N}(x, t)\right\|_{H^{m}(\Omega)}^{2} \leq C_{3} \int_{0}^{t} B(t)\left\|v^{N}(x, t)\right\|_{H^{m}(\Omega)}^{2} d t+C_{4}\left\|f e^{\lambda t}\right\|_{L_{2}\left(\Omega_{\infty}\right)}^{2} .
$$

By Gronwall-Bellman inequality, we have

$$
\left\|v^{N}(x, t)\right\|_{H^{m}(\Omega)}^{2} \leq C_{4}\left\|f e^{\lambda t}\right\|_{L_{2}\left(\Omega_{\infty}\right)}^{2} e^{C_{3} \int_{0}^{t} B(t) d t} .
$$

Thus,

$$
\left\|v^{N}(x, t)\right\|_{H^{m}(\Omega)}^{2} \leq C_{5}\left\|f e^{\lambda t}\right\|_{L_{2}\left(\Omega_{x}\right)}^{2},
$$

i.e.,

$$
e^{2 \lambda t}\left\|u^{N}(x, t)\right\|_{H^{\prime \prime}(\Omega)}^{2} \leq C_{5}\left\|f e^{\lambda t}\right\|_{L_{2}\left(\Omega_{\infty}\right)}^{2} .
$$


Hence,

$$
\left\|u^{N}(x, t)\right\|_{H^{m, 0}\left(\Omega_{\infty}\right)}^{2} \leq C_{6}\left\|f e^{\lambda t}\right\|_{L_{2}\left(\Omega_{\infty}\right)}^{2} .
$$

Multiplying (13) by $\frac{d\left(C_{\ell}^{N}(t)\right)}{d t}$, taking the sum in $\ell$ from 1 to $N$, and integrating in $t$ from 0 to $t$, we obtain

$$
\begin{aligned}
& \int_{\Omega_{l}}\left[u_{t}^{N} \overline{u_{t}^{N}}+\sum_{|p|,|q|=1}^{m}(-1)^{m+|p|} a_{p q} D^{q} u^{N} \overline{D^{p} u_{t}^{N}}+\lambda_{0} u^{N} \overline{u_{i}^{N}}\right] d x d t+ \\
& +(-1)^{m} \int_{\Omega_{l}}\left(\sum_{|p|=1}^{m} a_{p} D^{p} u^{N} \overline{u_{t}^{N}}+a_{0} u^{N} \overline{u_{t}^{N}}\right) d x d t=-\int_{\Omega_{i}} f \overline{u_{t}^{N}} d x d t .
\end{aligned}
$$

Hence,

$$
\begin{gathered}
2\left\|u_{i}^{N}\right\|_{L_{2}\left(\Omega_{l}\right)}^{2} \\
+\int_{\Omega}\left[\sum_{|p|,|q|=1}^{m}(-1)^{m+|p|} a_{p q}(x, t) D^{q} u^{N}(x, t) \overline{D^{p} u^{N}(x, t)}+\lambda_{0}\left\|u^{N}(x, t)\right\|^{2}\right] d x= \\
=\int_{\Omega_{l}} \sum_{|p|,|q|=1}^{m}(-1)^{m+|p|} \frac{\partial a_{p q}}{\partial t} D^{q} u^{N} \overline{D^{p} u^{N}} d x d t- \\
-(-1)^{m} 2 \operatorname{Re} \int_{\Omega_{l}}\left[\sum_{|p|=1}^{m} a_{p} D^{p} u^{N} \overline{u_{t}^{N}}+a_{0} u^{N} \overline{u_{i}^{N}}\right] d x d t-2 \operatorname{Re} \int_{\Omega_{l}} \overline{f u_{i}^{N}} d x d t .
\end{gathered}
$$

By Cauchy inequality and (2) we obtain

$$
\left\|u_{t}^{N}\right\|_{L_{2}\left(\Omega_{t}\right)}^{2}+\left\|u^{N}(x, t)\right\|_{H^{m}(\Omega)}^{2} \leq D_{1}\left\|u^{N}\right\|_{H^{m, 0}\left(\Omega_{l}\right)}^{2}+D_{2}\|f\|_{L_{2}\left(\Omega_{l}\right)}^{2} .
$$

Thus,

$$
\begin{aligned}
\left\|u_{i}^{N}\right\|_{L_{2}\left(\Omega_{L}\right)}^{2} & \leq D_{1}\left\|u^{N}\right\|_{H^{m, o}\left(\Omega_{t}\right)}^{2}+D_{2}\|f\|_{L_{2}\left(\Omega_{l}\right)}^{2} \Rightarrow\left\|u_{t}^{N}\right\|_{L_{2}\left(\Omega_{\infty}\right)}^{2} \leq \\
& \leq D_{1}\left\|u^{N}\right\|_{H^{m, 0}\left(\Omega_{\infty}\right)}^{2}+D_{2}\|f\|_{L_{2}\left(\Omega_{\infty}\right)}^{2} \leq \\
& \leq D_{1}\left\|u^{N}\right\|_{H^{m, \circ}\left(\Omega_{\infty}\right)}^{2}+D_{2}\left\|f e^{\lambda t}\right\|_{L_{2}\left(\Omega_{\infty}\right)}^{2} .
\end{aligned}
$$

According to (18), we obtain

$$
\left\|u_{t}^{N}\right\|_{L_{2}\left(\Omega_{\infty}\right)}^{2} \leq\left(C_{6} D_{1}+D_{2}\right)\left\|f e^{\lambda t}\right\|_{L_{2}\left(\Omega_{\infty}\right)}^{2} .
$$

Thus, (18), (21) imply

$$
\left\|u^{N}\right\|_{H^{m, 1}\left(\Omega_{\infty}\right)}^{2} \leq C\left\|f e^{\lambda t}\right\|_{L_{2}\left(\Omega_{\infty}\right)}^{2},
$$

where $C$ does not depend on $N$. 
Since the sequence of functions $\left\{u^{N}\right\}$ is uniformly bounded in $H^{m, 1}\left(\Omega_{\infty}\right)$, we can take a subsequence which is weakly convergent in $H^{m, 1}\left(\Omega_{\infty}\right)$ to some function $u(x, t)$. We will prove that $u(x, t)$ is the solution of (4)-(6). Since $u^{N}(x, 0)=0$ $\forall x \in \Omega$ and $u^{N}(x, t) \in \stackrel{\circ}{H}^{m, 1}\left(\Omega_{T}\right)$, it follows that $u(x, 0)=0, u(x, t) \in \stackrel{\circ}{H}^{m, 1}\left(\Omega_{T}\right)$. For any $T>0$, multiplying (13) by $d_{\ell}(t) \in H^{1}(0, T), d_{\ell}(T)=0$, taking the sum in $\ell$ from 1 to $N$ and integrating in $t$ from 0 to $t$, we obtain

$$
\begin{gathered}
\int_{\Omega_{T}} u_{t}^{N} \bar{\eta} d x d t+ \\
+(-1)^{m} \int_{\Omega_{r}}\left[\sum_{|p|,|q|=1}^{m}(-1)^{|p|} a_{p q} D^{q} u^{N} \overline{D^{p} \eta}+(-1)^{m} u^{N} \bar{\eta}\right] d x d t+ \\
+(-1)^{m} \int_{\Omega_{r}}\left[\sum_{|p|=1}^{m} a_{p} D^{p} u^{N} \bar{\eta}+a_{0} u^{N} \bar{\eta}\right] d x d t= \\
=-\int_{\Omega_{r}} f f \bar{\eta} d x d t .
\end{gathered}
$$

The above equality is true for any function $\eta \in M_{N}$, where $M_{N}$ is the set consisting of functions which have the form $\sum_{i=1}^{N} d_{i}(t) \varphi_{i}(x), d_{i}(t) \in H^{1}(0, T), d_{i}(T)=0$. Since $\left\{u^{N}\right\}$ is weakly convergent, passing to the limit as $N \rightarrow \infty$ in equality (23). We get

$$
\begin{gathered}
\int_{\Omega_{r}} u_{t} \bar{\eta} d x d t+(-1)^{m} \int_{\Omega_{T}}\left[\sum_{|p|,|q|=1}^{m}(-1)^{|p|} a_{p q} D^{q} u \overline{D^{p} \eta}+(-1)^{m} u \bar{\eta}\right] d x d t+ \\
+(-1)^{m} \int_{\Omega_{T}}\left[\sum_{|p|=1}^{m} a_{p} D^{p} u \bar{\eta}+a_{0} u \bar{\eta}\right] d x d t=-\int_{\Omega_{T}} f \bar{\eta} d x d t .
\end{gathered}
$$

Since $M=\bigcup_{N=1}^{\infty} M_{N}$ is dense in $\stackrel{\circ}{H}^{m, 0}\left(\Omega_{T}\right)$, it follows that (24) holds for any function $\eta \in \stackrel{\circ}{H}^{m, 0}\left(\Omega_{T}\right), \eta(x, T)=0$, i.e., $u(x, t)$ is a generalized solution of (4)(6). Moreover, the weak convergence of $\left\{u^{N}\right\}$ and (22) imply that

$$
\|u(x, t)\|_{H^{m, 1}\left(\Omega_{\infty}\right)}=\varliminf_{N \rightarrow \infty}\left\|u^{N}(x, t)\right\|_{H^{m, 1}\left(\Omega_{\infty}\right)} \leq C\left\|f e^{\lambda t}\right\|_{L_{2}\left(\Omega_{\infty}\right)} .
$$

The theorem is proved.

3. Asymptotic behaviour of solutions. We now consider the asymptotic behaviour as $t \rightarrow \infty$ of generalized solutions of the first boundary-value problem

$$
(-1)^{m-1} L(x, t, D) u-u_{t}=f(x, t),
$$

where $(x, t) \in \Omega_{\infty}$, such that 


$$
\begin{gathered}
\left.u(x, t)\right|_{t=0}=\varphi(x) \in \stackrel{\circ}{H}^{2 m}(\Omega), \\
\left.\frac{\partial^{j} u}{\partial \nu^{j}}\right|_{\Gamma_{\infty}}=0, \quad j=0, \ldots, m-1 .
\end{gathered}
$$

Theorem 3. Suppose that

1) $a_{p q}$ are continuous on $\bar{\Omega}_{\infty}$ whenever $|p|=|q|=m$;

2) $\left|\frac{\partial a_{p q}}{\partial t}, \frac{\partial a_{p}}{\partial t}\right| \leq \mu, 1 \leq|p|,|q| \leq m, \mu=$ const;

3) there exist $\lambda_{1}>\lambda_{2}>0$ and a function $B(t) \in L_{1}(0,+\infty)$ such that fe $e^{\lambda_{1} t} \in$ $\in L_{2}(0,+\infty)$, and

$$
\max \left\{\left|\frac{\partial a_{p q}}{\partial t}\right|,\left|\frac{\partial a_{p}}{\partial t}\right|,\left|a-(-1)^{m}\left(\lambda_{0}+\lambda_{2}\right) I\right|\right\} \leq B(t) \quad \forall(t, x) \in \Omega_{\infty} .
$$

Then problem $(25)-(27)$ has a generalized solution $u(x, t)$ in $H^{m, 1}\left(\Omega_{\infty}\right)$. Moreover, we have

$$
u(t, x)=\varphi(x) e^{-\lambda_{1} t}+o\left(e^{-\frac{\lambda_{2} t}{2}}\right) \quad \text { as } \quad t \rightarrow \infty .
$$

Proof. We set $v(x, t)=u(x, t)-\varphi(x) e^{-\lambda_{1} t}$. Then problem (25)-(27) can be written as

$$
(-1)^{m-1} L(x, t, D) v-v_{t}=f(x, t)+e^{-\lambda_{1} t}\left[(-1)^{m} L(x, t, D) \varphi-\lambda_{1} \varphi(x)\right],
$$

where $(x, t) \in \Omega_{\infty}$, such that

$$
\begin{gathered}
v(x, 0)=0 \quad \text { on } \quad \Omega, \\
\left.\frac{\partial^{j} v}{\partial \nu^{j}}\right|_{\Gamma_{\infty}}=0, \quad j=0, \ldots, m-1 .
\end{gathered}
$$

Consider $g(x, t)=f(x, t)+e^{-\lambda_{1} t}\left[(-1)^{m} L(x, t, D) \varphi-\lambda_{1} \varphi(x)\right]$. It is easy to see that $g e^{\lambda_{2} t} \in L_{2}\left(\Omega_{\infty}\right)$. Thus, by virtue of Theorem 2, problem (28)-(30) has a generalized solution $v(x, t) \in H^{m, 1}\left(\Omega_{\infty}\right)$. Furthermore, by the same proof for Theorem 2, as $N \rightarrow \infty$, inequality (16) implies that

$$
\begin{gathered}
\left\|e^{\lambda_{2} t} v(x, t)\right\|_{H^{m}(\Omega)}^{2} \leq C_{1}, \\
\left\|\left(e^{\lambda_{2} t} v(x, t)\right)_{t}\right\|_{L_{2}\left(\Omega_{\infty}\right)}^{2} \leq C_{2} .
\end{gathered}
$$

It follows from (31), (32) that

$$
\begin{gathered}
e^{\lambda_{2} t}\left[\|v(x, t)\|_{L_{2}(\Omega)}^{2}+\|D v(x, t)\|_{L_{2}(\Omega)}^{2}\right] \leq C_{1} e^{-\lambda_{2} t}, \\
\int_{0}^{\infty} e^{\lambda_{2} t}\left\|v_{t}(x, t)\right\|_{L_{2}(\Omega)}^{2} d t \leq
\end{gathered}
$$




$$
\begin{aligned}
& \leq \int_{0}^{\infty} e^{-\lambda_{2} t}\left\|\left(e^{\lambda_{2} t} v(x, t)\right)_{t}\right\|_{L_{2}(\Omega)}^{2} d t+ \\
& +\int_{0}^{\infty} \lambda_{2}^{2} e^{\lambda_{2} t}\|v(x, t)\|_{L_{2}(\Omega)}^{2} d t \leq C_{3} .
\end{aligned}
$$

Thus,

$$
\int_{0}^{\infty} e^{\lambda_{2} t}\left[\left\|v_{t}(x, t)\right\|_{L_{2}(\Omega)}^{2}+\|v(x, t)\|_{L_{2}(\Omega)}^{2}+\|D v(x, t)\|_{L_{2}(\Omega)}^{2}\right] d t<\infty .
$$

By virtue of the Nash inequality [10], this gives

$$
|v(x, t)| \leq C e^{-\frac{\lambda_{2}}{2} t} \quad \text { as } \quad t \rightarrow \infty .
$$

This implies that the generalized solution $u(x, t)$ of problem $(25)-(27)$ satisfies the inequality

$$
\left|u(x, t)-e^{-\lambda_{1} t} \varphi(x)\right| \leq C e^{-\frac{\lambda_{2}}{2} t} .
$$

The proof is completed.

1. Колдратьев B. A., Олейлик O. A. Краевые задачи для уравнения с частными пронзводными в негладких областях // Уепехи мат. наук. - 1983. - 38, вып. 2. - С. 3-76.

2. Назаров C. A., Пламелевский Б. А. Эллиптические задачи в областях с кусочно-гладкой границей. - М.: Наука, 1991. - 336 с.

3. Ладықселская О. А. Краевые задачи математической физики. - М.: Наука, 1973. - 407 с.

4. Нгуел Маиь Хуиг. О гладкости решения задачи Дирихле для гиперболических систем в областxх с коническими или угловыми точками // Докл. АН СССР. - 1998. - 362, № 2. - С. 161 - 164.

5. Ладызселская O.A. О нестационарных операторных уравнениях и их приложениях к линейным задачам математической физики // Мат. с6. - 1958. - 45, № 2. - С. 123-158.

6. Доаи Ваи Нгок. Асимптотика решений краевых задач для параболических уравнений второго порядка в окрестности угловой точки границы // Вестн. Моск. ун-та. Сер. мат. и мех. - 1984. - № 1. - C. 34-36.

7. Чan Зуй Хо, Эскил Г. И. Краевые задачи для параболических систем псевдодифференциальных уравнений // Докл. АН СССР. - 1971. - 198, № 1. - С. 50-53.

8. Apeфьев B. H., Копдратьев B. A. Асимптотическое поведение решений второй краевой задачи для нелинейных параболических уравнений // Дифференц. уравнения. - 1993. - 29, № 12. C. $2104-2116$.

9. Фикера $\Gamma$. Теоремы существования в теорин упругости. - М.: Мир, 1974. - 157 с.

10. Nash J. Continuity of solutions of parabolic and elliptic equations // Amer. J. Math. - 1958. - 80. P. $931-984$.

Received 14.09.2001 